\title{
Evaluation of the novel three-dimensional porous poly (L-lactic acid)/ nano- hydroxyapatite composite scaffold
}

\author{
Jianghong Huang ${ }^{\mathrm{a}, \mathrm{b}}$, Jianyi Xiong ${ }^{\mathrm{a}, \mathrm{b}}$, Jianquan Liu ${ }^{\mathrm{a}, \mathrm{b}}$, Weimin $\mathrm{Zhu}^{\mathrm{a}, \mathrm{b}}$, Jielin Chen ${ }^{\mathrm{a}, \mathrm{b}}$, Li Duan ${ }^{\mathrm{a}, \mathrm{b}}$, \\ Jufeng Zhang ${ }^{\mathrm{a}, \mathrm{b}}$ and Daping Wang, ${ }^{\mathrm{a}, \mathrm{b}, *}$ \\ ${ }^{a}$ National Key Orthopedic Department, Shenzhen Second People's Hospital, Guangdong Province, \\ China \\ ${ }^{b}$ Shenzhen Tissue Engineering Key Laboratory, GuangDong Province, China
}

\begin{abstract}
To determine the optimal ratio of nano-hydroxyapatite (n-HA) to polylactic acid (PLLA) in the novel three-dimensional porous PLLA/n-HA composite scaffolds, low-temperature rapid prototyping technology was employed to fabricate the composite materials with different n-HA contents. Mechanical properties and degradation behaviors of the composites were examined, and the scaffold microstructure and n-HA dispersion were observed by scanning electron microscope (SEM). Mechanical tests demonstrated that the tensile strength of the composite material gradually decreased with an increase in $\mathrm{n}-\mathrm{HA}$ content. When the $\mathrm{n}-\mathrm{HA}$ content reached $20 \mathrm{wt} \%$, the bending strength of the composite material peaked at $138.5 \mathrm{MPa}$. SEM images demonstrated that the optimal content of $\mathrm{n}-\mathrm{HA}$ was $20 \mathrm{wt} \%$ as the largest interconnected pore size that can be seen, with a porosity as high as $80 \%$. In vitro degradation experiments demonstrated that the $\mathrm{pH}$ value of the material containing solution gradually decreased in a time-dependent manner, with a simultaneous weakening of the mechanical properties. In vitro study using rat osteoblast cells showed that the composite scaffolds were biocompatible; the $20 \mathrm{wt} \% \mathrm{n}$-HA scaffold offered particular improvement to rat osteoblast cell adhesion and proliferation compared to other compositions. It was therefore concluded that $20 \mathrm{wt} \% \mathrm{n}-\mathrm{HA}$ is the optimal nano-hydroxyapatite (n-HA) to polylactic acid (PLLA) ratio, with promise for bone tissue engineering.
\end{abstract}

Keywords: Three-dimensional porous, polylactic acid, nano-hydroxyapatite, rapid prototyping

\section{Introduction}

Artificial bone substitute materials, as an alternative to autografts and allografts, have attracted much attention in recent years. An ideal material has not yet been developed for the repair of bone defects. Generally, bone graft substitutes from one phase of a material have drawbacks, and can barely satisfy the requirements for artificial bone grafts [1]. Nano-hydroxyapatite (n-HA), similar to the inorganic component of native bone tissue, has great biomechanical properties and is considered to be a promising biomaterial for bone repair; however, its high fragility limits its applications in bone defect repair. Poly-lactic acid (PLLA) is a biocompatible and biodegradable polymer which has been widely used in bone tissue engineering. However, PLLA alone lacks osteoinductivity and cannot provide a suitable environment for osteoblast or osteoblast-like cells to deposit minerals.

Cijun Shuai et al. [2] fabricated an n-HA scaffold using selective laser sintering with added to

\footnotetext{
* Address for correspondence: Daping Wang, National Key Orthopedic Department, Shenzhen Second People's Hospital, Guangdong Province, China. Tel.: 13602511630; Fax: 0755-83792752; E-mail: dapingwang1963@qq.com.
} 
improve its mechanical strength. Minli Zhao et al. [3] and Xiuyan Li et al. [4] developed a PLLA/n-HA scaffold by electrospinning and melt electrospinning, respectively. Although the scaffolds mimicked the natural ECM at nanoscale, the scaffold pore sizes and porosity cannot be controlled and cannot definitively meet the needs of bone remodeling. Yingchun Liang et al. [5] fabricated the PLLA/n-HA scaffold by low-temperature rapid prototyping (LRP) technology, yet the optimal proportion of the materials was undetermined. LRP technology is a rapid prototyping technology to fabricate three dimensional scaffolds. It has no negative effect on the character of materials and allows the precise control of pore size and porosity of the scaffold through the use of a designed model on the CAD system.

In previous studies, we employed the LRP technology to fabricate novel three-dimensional porous PLLA/n-HA composite scaffolds with various PLLA/n-HA ratios [6]. The excellent processability, biocompatibility and degradability of PLLA and the great osteoconductivity of n-HA, makes the porous PLLA/n-HA composite a promising bone substitute material for new bone formation. In this study, we performed mechanical testing, a scaffold degradation study, SEM imaging and in vitro study to determine the optimal PLLA/n-HA ratio for scaffold fabrication.

\section{Materials and methods}

\subsection{Preparation of poly-L-lactic acid and $n-H A$}

A high purity L-lactide (99.95\%) was prepared by vacuum distillation technique and washing-recrystallization purification. Lactic acid (Jiangxi Musashino Bio Chemical Co., Ltd) and stannous octanoate (Sigma-Aldrich, U.S.) were used as the raw material and as the catalyst, respectively. Poly-L-lactic acid (PLLA) was then synthesized by in situ polymerization using L-lactide and stannous octoate (reaction temperature: $150^{\circ} \mathrm{C}$; reaction time: 24 hours) [7]. The molecular weight of the obtained PLLA $(\mathrm{Mn}=400,000)$ was related to the purity of lactide, polymerization temperature and vacuum degree. The n-HA powder was prepared by chemical precipitation using diammonium phosphate, calcium nitrate and ammonia (Shenzhen Heaven) as the raw materials. According to the chemical formula [8] $5 \mathrm{Ca}\left(\mathrm{NO}_{3}\right)_{2} \cdot 4 \mathrm{H}_{2} \mathrm{O}+3\left(\mathrm{NH}_{4}\right) 2 \mathrm{HPO}_{4}+4 \mathrm{NH}_{3} \cdot \mathrm{H}_{2} \mathrm{O} \rightarrow \mathrm{Ca} 5\left(\mathrm{PO}_{4}\right)_{3} \mathrm{OH}+10 \mathrm{NH}_{4} \mathrm{NO}_{3}$ $+3 \mathrm{H}_{2} \mathrm{O}$, the n-HA powder was milled for 24 hours in a ceramic jar after the white sedimentation cake had dried. The n-HA powder was calcined at $850^{\circ} \mathrm{C}$ for 1 hour to increase degree of crystallinity. Well-dispersed and bar-shaped n-HA particles were then observed; particle size was approximately $30-50 \mathrm{~nm}$ in diameter and approximatley $30-100 \mathrm{~nm}$ in length, with fine crystallization property as

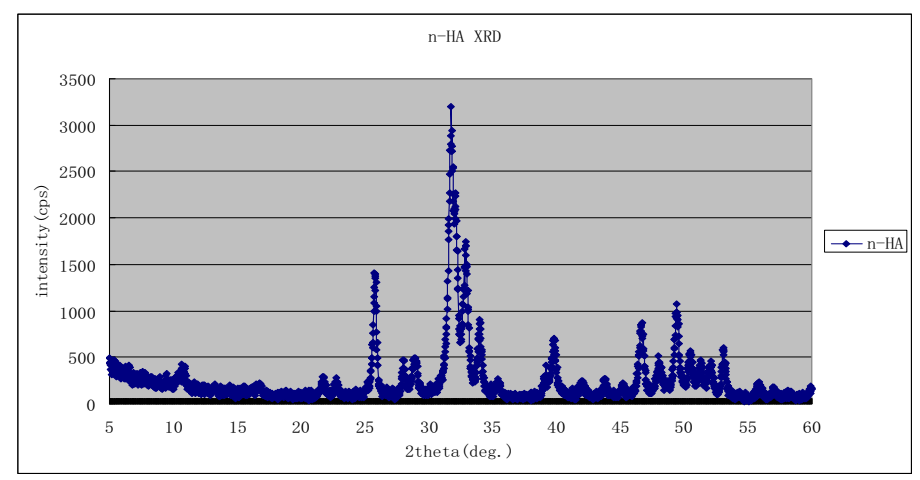

Fig. 1. XRD curves of n-HA powder. 


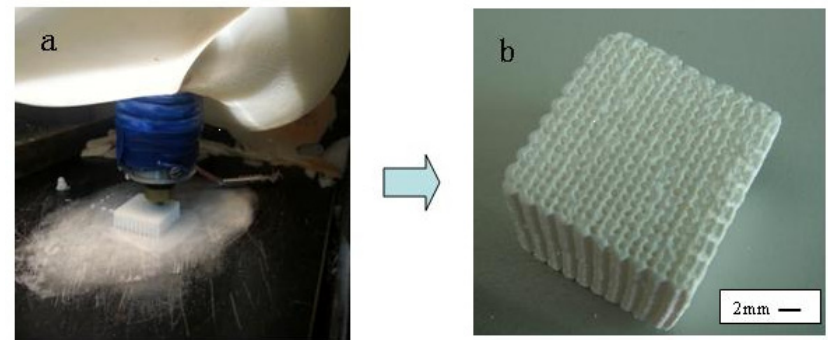

Fig. 2. The fabrication of the 3D print PLLA/n-HA composite scaffold via LRP (a) the optical image of the three-dimensional porous PLLA/n-HA scaffold; (b) size of the sample $=25 \times 25 \times 25 \mathrm{~mm}^{3}$.

shown in Figure 1. Preparations of L-lactide and poly-L-lactic acid as well as the nano-hydroxyapatite were developed in the Second People's Hospital of Shenzhen.

\subsection{Preparation of three-dimensional porous PLLA/n-HA composites}

The PLLA solution was obtained by dissolving 25\% (w/v) PLLA in 1, 4-dioxane; solutions with different $\mathrm{n}$-HA weight percentages $(0,10 \mathrm{wt} \%, 20 \mathrm{wt} \%, 30 \mathrm{wt} \%, 40 \mathrm{wt} \%)$ were prepared by adding different amounts of n-HA to the PLLA solution. After proper mixing, homogenous dispersion of n-HA particles could be observed in the solution. As previously described [9], the three-dimensional scaffold was printed by a LRP device, controlled by computer software (With this software that can design material 3D model we want, by regulating the parameters of the computer print out the model). The LRP device provided by the Shenzhen Institutes of Advanced Technology Chinese Academy of Sciences Centre was used to fabricate the three-dimensional porous PLLA/n-HA composites. The parameters were set as follows: chamber temperature: $-20^{\circ} \mathrm{C}$; nozzle temperature: $17^{\circ} \mathrm{C}$; nozzle diameter: $0.3 \mathrm{~mm}$; nozzle speed: $15.0 \mathrm{~mm} / \mathrm{s}$; extrusion speed $1.80 \mathrm{~mm} / \mathrm{s}$; strand distance: $1 \mathrm{~mm}$. As shown in Figure 2(a), the three dimensional porous scaffold was printed layer by layer under the computer's control. One representative PLLA/n-HA composite sample is shown in Figure 2(b).

\subsection{Characterization of composite materials}

\subsubsection{Mechanical testing}

Tensile strength, flexural strength and flexural modulus testing were performed to optimize the scaffold with proper mechanical properties to mimic natural bone; measurements were obtained through the use of the Sansi electronic universal testing machine CTM4000 and bending strength was obtained by the three-point bending method. The span length was $20 \mathrm{~mm}$ and the loading rate was 5.00 $\mathrm{mm} / \mathrm{min}$.

\subsubsection{Porosity test}

The dimensions of the samples were measured using a Vernier caliper, for the calculation of the volumes $(\mathrm{V})$ of the scaffolds. The dry scaffolds were weighed $\left(\mathrm{M}_{1}\right)$, submerged in absolute ethanol with a density of $r$ for 3 hours, and weighed again $\left(M_{2}\right)$. The porosity was calculated by $\left(M_{2}-M_{1}\right) / r / V$, $(n=10)$.

\subsubsection{Microstructure observation}

The three-dimensional porous PLLA/n-HA composite scaffold was prepared by brittle fracture under low temperatures and coated with gold for SEM imaging. The microstructure of the sample was 
then observed by a field emission scanning electron microscope (Tescan, Czech) provided by the Research Institute of Tsinghua University in Shenzhen Micro/Nano Engineering Key Lab.

\subsubsection{In vitro degradation study}

The sample was vacuum-dried to a constant weight $\left(\mathrm{W}_{0}\right)$ and then placed in tubes containing phosphate buffered saline (PBS, $\mathrm{PH}=7.4, \mathrm{n}=10)$. The tubes were shaken in an incubator $\left(37^{\circ} \mathrm{C} \pm 1\right)$. The $\mathrm{pH}$ value of the solution with the degradation products was measured with a $\mathrm{pH}$ meter, and composite size was measured after washing with distilled water at weeks 2, 4, 6, 8 and 10. All samples were dried to constant weight $\left(\mathrm{W}_{\mathrm{T}}\right)$ followed by examination of tensile strength, flexural strength and flexural modulus.

\subsubsection{Cell attachment and proliferation}

Rat osteoblast cells were isolated by enzyme digestion [10]. Cell adhesion was evaluated by the cell count method. Rat osteoblasts at passage 3 were seeded on a sample $(10 \mathrm{~mm} \times 10 \mathrm{~mm} \times 0.5 \mathrm{~mm})$ at a cell density of $5 \times 10^{5} / \mathrm{mL}(\mathrm{n}=10)$, followed by incubation at $37^{\circ} \mathrm{C}$ for 12 hours in humidified $5 \% \mathrm{CO}_{2}$. At the end of the incubation period, the solutions containing the unattached cells were discarded and each solution containing well-attached cells was washed three times with PBS. Cells were detached by trypsin/EDTA and counted under the optical microscope. Cell adhesion rate was calculated by the following equation: cell adhesion rate $=($ adherent cells/the total number of seeded cells $) \times 100 \%$. For the cell proliferation assay, the study was conducted on days 1, 3, 5, 7, 9 and 12. The medium was removed from the 24-well plate at each time point, followed by the addition of fresh medium containing $10 \%$ CCK- 8 for 3 hours of incubation. The absorbance then was measured at a wavelength of 450nm using a microplate reader (Model 550; Bio-Rad).

\subsection{Statistical analysis}

Statistical calculations were performed using SPSS software. The significance of difference was analyzed by t-test; a $p$-value $<0.05$ was considered statistically significant.

\section{Results and discussion}

3.1. Comparison of the mechanical properties of the three-dimensional porous PLLA/n-HA composites with different $n$-HA contents

As observed in Table 1, the tensile-strength and flexure modulus decreased linearly with an increase in n-HA content in the composite. The bending strength of the composite with $20 \mathrm{wt} \% \mathrm{n}-\mathrm{HA}$ content was $138.5 \mathrm{MPa}$, the highest of all examined compositions. The bending strength of the composite scaffold decrease below that of the PLLA and $20 \mathrm{wt} \% \mathrm{n}$-HA scaffold when the n-HA content exceeded $30 \mathrm{wt} \%$.

\subsection{Observation of the fracture surface of PLLA/n-HA composite scaffolds}

SEM images of the scaffold fracture surfaces are shown in Figure 3 and observed in Table 2. T PLLA was rough, containing small pores with a diameter of approximately $1 \mu \mathrm{m}$. The porosity was the fracture surface of the pure approximately $55 \%$, as demonstrated by Figure 3(a). After the addition of 
$10 \mathrm{wt} \% \mathrm{n}-\mathrm{HA}$, the groove on the fracture surface became larger than that of pure PLLA, as shown in Figure 3(b); larger pores were observed and the porosity increased to $60 \%$. The composite scaffold became porous, with the pores of approximately $10 \mu \mathrm{m}$ in diameter becoming interconnected, as demonstrated in Figure 3(c). Compared to the scaffold with $20 \mathrm{wt} \% \mathrm{n}-\mathrm{HA}$ content, its porosity increased by $20 \%$. When the n-HA mass fraction exceeded $20 \mathrm{wt} \%$, the fracture surface gradually developed larger grooves and smaller pores, as shown in Figures 3(d) and 3(e). Moreover, their porosity decreased to less than $70 \%$.

\subsection{Time-course changes of $\mathrm{pH}$ value and weight loss ratio}

As shown in Figure 4(a), during the first 8 weeks the $\mathrm{pH}$ value of the sample solution gradually declined in a time-dependent manner. The $\mathrm{pH}$ value decreased more slowly with a parallel increase of $\mathrm{n}$-HA content in the composite. The quantification of $\mathrm{pH}$ change is 6.3 , decreasing to 5.2 as the degradation time increased. For all groups, $\mathrm{pH}$ values showed a slight increase at week 10 compared to week 8. As shown in Figure 4(b), the weight loss ratio of the sample increased as the degradation time increased. The weight loss rate was highest until 6 weeks, at which point the rate of change decreased.

Table 1

The mechanical properties of three-dimensional porous PLLA/n-HA scaffolds $(\overline{\mathbb{E}} \pm \mathrm{S}, \mathrm{n}=10)$

\begin{tabular}{llll}
\hline n-HA mass fraction (wt $\%)$ & Tensile strength (Mpa) & Bending strength (Mpa) & Flexure modulus (Gpa) \\
\hline 0 & $42.5 \pm 0.6$ & $122.8 \pm 0.3$ & $2.8 \pm 0.1$ \\
10 & $38.6 \pm 0.5$ & $131.9 \pm 0.6$ & $3.1 \pm 0.1$ \\
20 & $35.1 \pm 0.6^{*}$ & $138.6 \pm 1.1^{*}$ & $3.5 \pm 0.1^{*}$ \\
30 & $29.2 \pm 0.4$ & $119.1 \pm 0.9$ & $3.8 \pm 0.1$ \\
\hline 40 & $23.2 \pm 0.5$ & $112.5 \pm 1.2$ & $3.9 \pm 0.1$ \\
\hline
\end{tabular}

Note: $* P$-value $\leq 0.05$ was considered statistically significant.

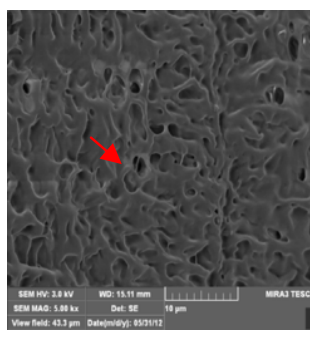

a

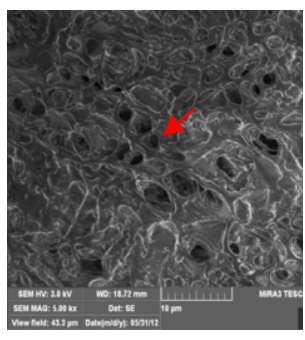

$\mathrm{b}$

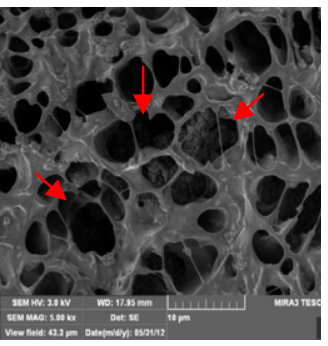

c

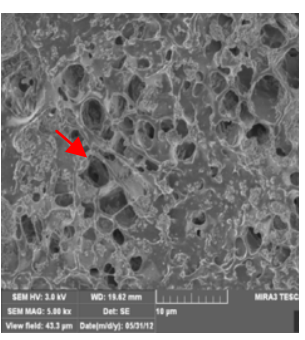

d

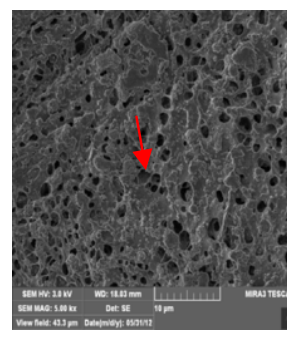

e

Fig. 3. SEM images of fracture surfaces of the three-dimensional porous PLLA/n-HA composites, with a scale bar of $10 \mu \mathrm{m}$. (a) PLLA; (b) $10 \mathrm{wt} \% \mathrm{n}-\mathrm{HA}$; (c) $20 \mathrm{wt} \% \mathrm{n}-\mathrm{HA}$; (d) $30 \mathrm{wt} \% \mathrm{n}-\mathrm{HA}$; (e) $40 \mathrm{wt} \% \mathrm{n}-\mathrm{HA}$.

Table 2

The pore size and porosity of composite scaffolds $(\bar{x} \pm S, n=10)$

\begin{tabular}{llll}
\hline $\mathrm{n}$-HA content $(\mathrm{wt} \%)$ & Pore size $(\mu \mathrm{m})$ & Internal pore size $(\mu \mathrm{m})$ & Porosity $(\%)$ \\
\hline 0 & 271 & $2.6 \pm 0.3$ & $55.3 \pm 0.6$ \\
10 & 336 & $4.1 \pm 0.1$ & $68.5 \pm 0.2$ \\
20 & 392 & $9.3 \pm 0.5$ & $85.1 \pm 0.9$ \\
30 & 354 & $5.7 \pm 0.7$ & $76.3 \pm 0.6$ \\
\hline 40 & 318 & $3.1 \pm 0.3$ & $72.2 \pm 0.5$ \\
\hline
\end{tabular}


After 10 weeks, all samples demonstrated a weight loss of $11 \%$ in total.

\subsection{Time-course changes of mechanical properties during the degradation process}

The tensile strength, bending strength and bending modulus of the composites decreased in a time-dependent manner during the degradation process, as demonstrated by Figures 4(c), 4(d) and 4(e).

\subsection{Biocompatibility of $3 D$ composite PLLA/n-HA scaffolds}

\subsubsection{Cell adhesion result}

As shown in Table 3, the adhesion rate of the cells on $20 \mathrm{wt} \% \mathrm{n}-\mathrm{HA}$ is $75.70 \pm 1.21 \%$ after 12 hours, much higher than those of other groups $(p<0.05)$.
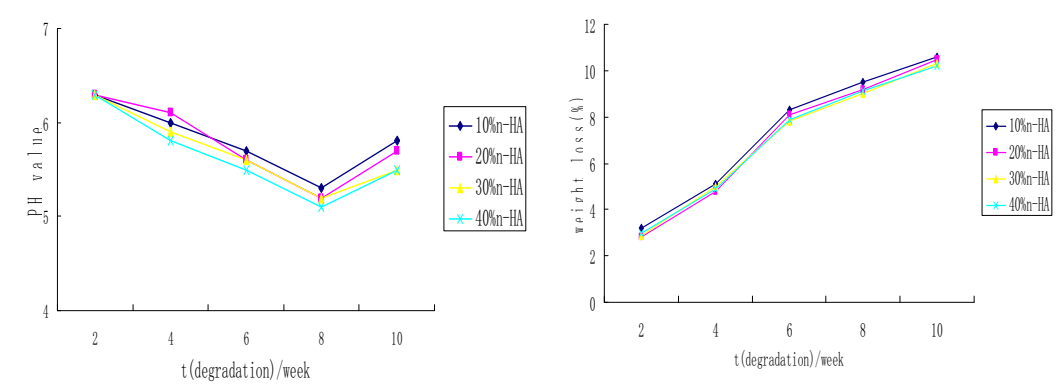

b

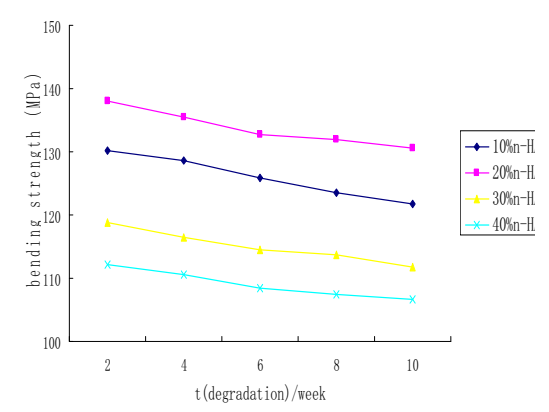

d

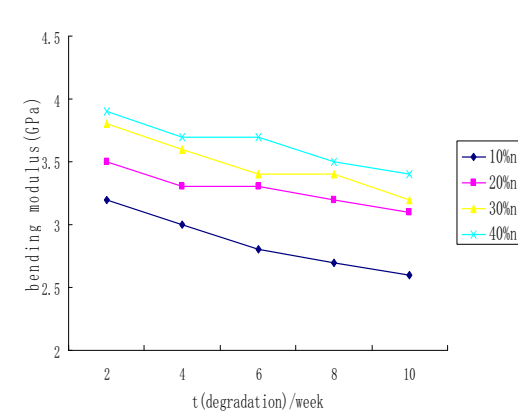

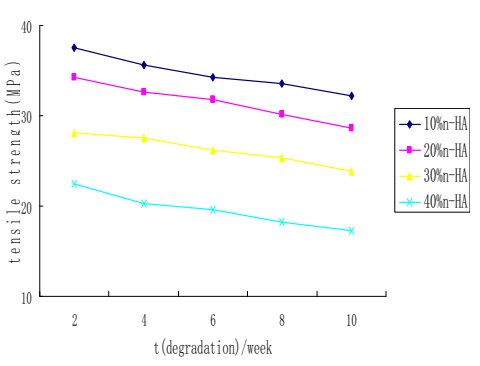

c

Fig. 4. (a) Time-related changes of $\mathrm{pH}$ value of solution containing degradation products; (b) Change in weight loss of samples as the degradation time progressed; (c) The time-course changes in tensile strength; (d) The time-course changes of bending strength; (e) The time-course changes in bending modulus.

Table 3

Cell adhesion rate to PLLA/n-HA scaffolds $(\bar{x} \pm \mathrm{S}, \quad \mathrm{n}=10)$

\begin{tabular}{ll}
\hline n-HA content $(\%)$ & Cell adhesion rate $(\%)$ \\
\hline 0 & $62.50 \pm 1.32$ \\
10 & $68.30 \pm 1.25$ \\
20 & $79.70 \pm 1.17$ \\
30 & $72.20 \pm 1.28$ \\
\hline 40 & $70.10 \pm 1.22$ \\
\hline
\end{tabular}




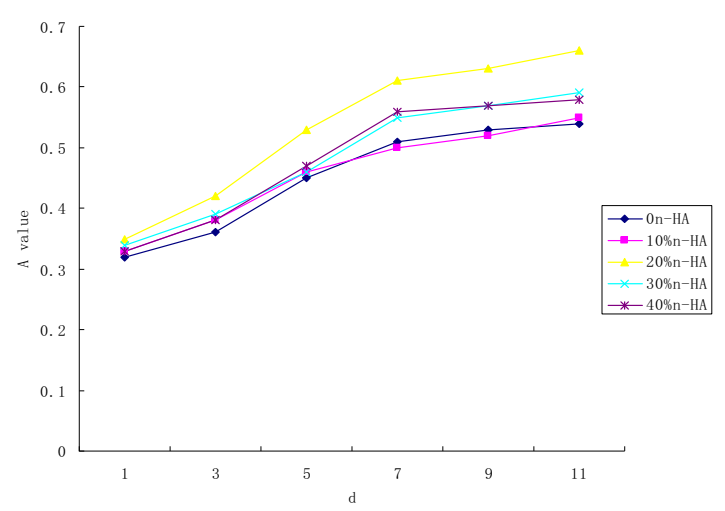

Fig. 5. The proliferation of rat osteoblast cells on scaffolds containing different amounts of n-HA.

\subsubsection{Cell proliferation result}

As shown in Figure 5, the cells on samples proliferated steadily as the culture time increased. At day 1 , the data showed that the number of cells on the $20 \mathrm{wt} \% \mathrm{n}-\mathrm{HA}$ scaffold was higher than that of other groups, which might be attributed to a higher number of adherent cells. Along with the increasing time of cell culture, the cells on $20 \mathrm{wt} \% \mathrm{n}$-HA scaffold showed in significantly higher numbers than those of other groups $(p<0.05)$. Studies showed that scaffold with suitable pore size and porosity had a positive effect on cell attachment and proliferation. In our case, the scaffold containing $20 \mathrm{wt} \% \mathrm{n}-\mathrm{HA}$ had much larger interconnected pores, providing more spaces for cells to grow and migrate.

\subsection{Discussion}

Pure n-HA is well known for its good osteoconductivity, fine biocompatibility and chemostability [11]. However, its high fragility makes it a rare candidate for the repair of weight-bearing sites. Polymeric materials with fine biocompatibility and degradability gradually become ideal orthopedic fixation materials. PLLA is one of the most widely used degradable materials and was approved by the FDA for application in bone defect repair and internal fixation of fractures. There is no need to reopen or remove materials from the internal fracture fixator made from PLLA after implantation, which avoids the need for multiple surgeries and simplifies the operative procedure. However, the breaking elongation percentage of pure PLLA is low, and reveals no osteoconductivity. Therefore, the composite PLLA/n-HA is posited as a more beneficial ideal for artificial bone material.

The PLLA/n-HA composite scaffold can be fabricated by many methods including film casting [12], particulate leaching [13] and gas foaming [14]. Although these methods are convenient, they fail to accurately control the pore size and porosity of the scaffold. By the aid of computer software, LRP is an advanced technology to fabricate scaffolds with controlled pore size and porosity. In the present study, PLLA/n-HA composite scaffold fabricated by LRP have regular and large pores and high porosity.

Experiments in mechanics and obtained SEM images in the present study demonstrated numerous pores and tracks to be observed in the fracture surfaces with the addition of n-HA. When the content of $\mathrm{n}-\mathrm{HA}$ reached $20 \mathrm{wt} \%$, most $\mathrm{n}$-HA molecules were packaged by the materials, forming a tight integrated interface to help conduct stress and prevent the crackle extension, thus improving the elasticity of the materials. When the n-HA weight exceeded $30 \mathrm{wt} \%$, n-HA showed potential to agglomerate and negatively affect the mechanical properties of the scaffold. Moreover, the porosity of 
$20 \mathrm{wt} \% \mathrm{n}-\mathrm{HA}$ was the highest to possess large enough pores for cells to grow in [15]. If many n-HA molecules could not be packaged by the materials, the relatively tight integrated interface was broken and the strength and elasticity were decreased from our previous studies.

An in vitro degradation test demonstrated the effectiveness of $n-H A$ to reduce the acidity created by PLLA degradation, which could help reduce or avoid aseptic inflammation and improve biocompatibility. With the healing of the fracture, the PLLA/n-HA composites would gradually degrade in vivo, facilitating the penetration of the cells and later new bone formation [16].

Cell adhesion rate and proliferation on scaffold indicate the biocompatibility of the scaffold, which in turn supports the proliferation, migration and differentiation of cells in the process of bone repair. Cells in scaffold secrete ECM and deposit minerals to form new bone [17]. The in vitro results demonstrated that scaffolds with $20 \mathrm{wt} \% \mathrm{n}$-HA content enhanced cell adhesion and proliferation when compared to other groups, which may be contributed to the component and/or to the porous structure of the scaffold [18]. The n-HA was hydrophilic and a better bioactive agent than PLLA, which may promote cell adhesion. Additionally, the pore size of scaffold was approximately $400 \mu \mathrm{m}$, which may be beneficial to cell adhesion, migration and proliferation. Studies have previously showed that the microenvironment of cell growth played an important role in cell proliferation. Acidic condition might result in the cell detachment from the scaffold. In our case, during the degradation of the scaffold containing $20 \mathrm{wt} \% \mathrm{n}-\mathrm{HA}$, the $\mathrm{pH}$ value of the solution was higher than that of other groups, which might offer another potential explanation for enhanced cell proliferation.

\section{Conclusion}

In this study, novel three-dimensional porous PLLA/n-HA composites with different PLLA/n-HA ratios were fabricated using low-temperature rapid prototyping technology, with the comparison and the evaluation of their mechanical properties, microstructures, degradation behaviors and bioactivity. The study showed that the composite with $20 \mathrm{wt} \% \mathrm{n}-\mathrm{HA}$ possessed improved mechanical properties and dissolution stability compared to other compositions. Acid derived from PLLA degradation might be neutralized by n-HA, thereby reducing the acidoid accumulation. In vitro study revealed that the 20 wt $\%$ n-HA scaffold enabled more rat osteoblast cells to adhere and proliferate. Therefore, it was concluded that the PLLA/n-HA scaffold containing $20 \mathrm{wt} \% \mathrm{n}-\mathrm{HA}$ content was the optimal composition, making it a promising material for bone tissue repair and internal fixation of fractures.

\section{Acknowledgments}

This work was supported in part by Shenzhen Key Laboratory Upgrading Project (No. CXB201104220049A), Shenzhen R\&D funding project (No. CXZZ20120614160234842, ZDSY20120614154551201, JCYJ20130401113330839, CXZZ20130321152713220 and CXZZ20140813160132596).

\section{References}

[1] J. Russias, E. Saiz, R.K. Nalla and A.P. Tomsia, Microspheres as building blocks for hydroxyapatite/polylactide biodegradable composites, Journal of Materials Science 41 (2006), 5127-5133. 
[2] Cijun Shuai, Yi Nie, Chengde Gaol, et al., Poly (l-lactide acid) improves complete nano- hydroxyapatite bone scaffolds through the microstructure rearrangement, Electronic Journal of Biotechnology 15 (2012), 345-368.

[3] Min-li Zhao, Gang Sui, Xu-liang Deng, et al., PLLA/HA electrospin hybrid nanofiber scaffolds: Morphology, in vitro degradation and cell culture potential, Advanced Materials Research 12 (2006), 243-246.

[4] Xiuyan Li, Huichao Liu, Jiaona Wang, et al., Preparation and characterization of PLLA/nHA nonwoven mats via laser melt electrospinning, Materials Letters 15 (2012), 103-106.

[5] Yingchun Liang, Xiongfei Zheng, Wenjie Zhai, et al., 3D PLLA/nano-hydroxyapatite scaffolds with hierarchical porous structure fabricated by low-temperature deposition manufacturing, Journal of Wuhan University of Technology-Mater 8 (2012), 265-269.

[6] T. Wang, L.C. Chow, S.A. Frukhtbeyn, et al., Improve the strength of PLA/HA composite through the use of surface initiated polymerization and phosphonic acid coupling agent, Journal of Research of the National Institute of Standards and Technology 116 (2011), 785-796.

[7] Z. Hong, P. Zhang, C. He, et al., Nano-composite of poly(l-lactide) and surface grafted hydroxyapatite: Mechanical properties and biocompatibility, Biomaterials 26 (2005), 6296-6304.

[8] Hai-lin Yang, Jing Li, Hua-chan Fang, et al., Synthesis, characterization and biological activity in vitro of $\mathrm{FeCrAl}(\mathrm{f}) / \mathrm{HA}$ asymmetrical biological functionally gradient materials, Journal of Central South University 21 (2014), 447-453.

[9] Mei-jian He, Da-ping Wang, Jiang-hong Huang, et al., Preparation and properties of hydrophilic modified artificial bone scaffold using low-temperature rapid prototyping, Chinese Journal of Tissue Engineering Research 18 (2014), 1824-1830.

[10] Xiao-feng Li, Jin-min Zhao, Wei Su, et al., Primary culture and identification of rat osteoblasts, Journal of Clinical Rehabilitative Tissue Engineering Research 15 (2011), 990-994.

[11] S.J. Kalita and H.A. Bhatt, Nanocrystalline hydroxyapatite doped with magnesium and zinc: Synthesis and characterization, Materials Science and Engineering C 27 (2007), 837-848.

[12] T. Tian, D. Jiang, J. Zhang, et al., Fabrication of bioactive composite by developing PLLA onto the framework of sintered HA scaffold, Materials Science \& Engineering C 28 (2008), 51-56.

[13] T. Kasuga, Y. Ota, M. Nogami, et al., Preparation and mechanical properties of polylactic acid composites containing hydroxyapatite fibers, Biomaterials 22 (2001), 19-23.

[14] Claire Delabarde, Christopher J.G. Plummer and Pierre-Etienne Bourban, Biodegradable polylactide/hydroxyapatite nanocomposite foamscaffolds for bone tissue engineering applications, Journal of Materials Science: Materials in Medicine 23 (2012), 1371-1385.

[15] L. Varila, T. Lehtonen and J. Tuominen, In vitro behaviour of three biocompatible glasses in composite implants, Journal of Materials Science 23 (2012), 2425-2435.

[16] A. Obata, T. Hotta, T. Wakita, et al., Electrospun microfiber meshes of silicon-doped vaterite/poly(lactic acid) hybrid for guided bone regeneration, Acta Biomater 6 (2010), 1248-1257.

[17] B.D. Boyan, T.W. Hummert, D.D. Dean, et al., Role of material surfaces in regulating bone and cartilage cell response, Biomaterials 17 (1996), 137-146.

[18] C.M. Stanford, M. Solursh and J.C. Keller, Significant role of adhesion properties of primary osteoblast-like cells in early adhesion events for chondroitin sulfate and dermatan sulfate surface molecules, Journal of Biomedical Materials Research 47 (1999), 345-352. 